\title{
Front Matter: Volume 7379
}

, "Front Matter: Volume 7379," Proc. SPIE 7379, Photomask and NextGeneration Lithography Mask Technology XVI, 737901 (24 April 2009); doi: $10.1117 / 12.833610$

SPIE. Event: Photomask and NGL Mask Technology XVI, 2009, Yokohama, Japan 


\title{
PROCEEDINGS OF SPIE
}

\section{Photomask and Next-Generation Lithography Mask Technology XVI}

\author{
Kunihiro Hosono \\ Editor \\ 8-10 April 2009 \\ Yokohama, Japan \\ Sponsored by \\ PMJ Photomask Japan \\ BACUS \\ SPIE \\ Published by \\ SPIE
}

Volume 7379 
The papers included in this volume were part of the technical conference cited on the cover and title page. Papers were selected and subject to review by the editors and conference program committee. Some conference presentations may not be available for publication. The papers published in these proceedings reflect the work and thoughts of the authors and are published herein as submitted. The publisher is not responsible for the validity of the information or for any outcomes resulting from reliance thereon.

Please use the following format to cite material from this book:

Author(s), "Title of Paper," in Photomask and Next-Generation Lithography Mask Technology XVI, edited by Kunihiro Hosono, Proceedings of SPIE Vol. 7379 (SPIE, Bellingham, WA, 2009) Article CID Number.

ISSN 0277-786X

ISBN 9780819476562

Published by

SPIE

P.O. Box 10, Bellingham, Washington $98227-0010$ USA

Telephone +1 3606763290 (Pacific Time) · Fax +1 3606471445

SPIE.org

Copyright (C) 2009, Society of Photo-Optical Instrumentation Engineers

Copying of material in this book for internal or personal use, or for the internal or personal use of specific clients, beyond the fair use provisions granted by the U.S. Copyright Law is authorized by SPIE subject to payment of copying fees. The Transactional Reporting Service base fee for this volume is $\$ 18.00$ per article (or portion thereof), which should be paid directly to the Copyright Clearance Center (CCC), 222 Rosewood Drive, Danvers, MA 01923. Payment may also be made electronically through CCC Online at copyright.com. Other copying for republication, resale, advertising or promotion, or any form of systematic or multiple reproduction of any material in this book is prohibited except with permission in writing from the publisher. The CCC fee code is 0277-786X/09/\$18.00.

Printed in the United States of America.

Publication of record for individual papers is online in the SPIE Digital Library.

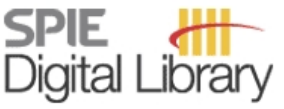

SPIEDigitalLibrary.org

Paper Numbering: Proceedings of SPIE follow an e-First publication model, with papers published first online and then in print and on CD-ROM. Papers are published as they are submitted and meet publication criteria. A unique, consistent, permanent citation identifier (CID) number is assigned to each article at the time of the first publication. Utilization of CIDs allows articles to be fully citable as soon they are published online, and connects the same identifier to all online, print, and electronic versions of the publication. SPIE uses a six-digit CID article numbering system in which:

- The first four digits correspond to the SPIE volume number.

- The last two digits indicate publication order within the volume using a Base 36 numbering system employing both numerals and letters. These two-number sets start with 00, 01, 02, 03, 04 , 05, 06, 07, 08, 09, OA, OB ... 0Z, followed by 10-1Z, 20-2Z, etc.

The CID number appears on each page of the manuscript. The complete citation is used on the first page, and an abbreviated version on subsequent pages. Numbers in the index correspond to the last two digits of the six-digit CID number. 


\section{Contents}

xiii Symposium Committees

xv Conference Committees

\section{INVITED SESSION}

737902 Litho/mask strategies for 32-nm half-pitch and beyond: using established and adventurous tools/technologies to improve cost and imaging performance (Keynote Paper) [7379-01]

B. J. Lin, Taiwan Semiconductor Manufacturing Co. Ltd. (Taiwan)

\section{MATERIAL AND PROCESS I}

737906 The novel plasma etching process for defect reduction in photomask fabrication [7379-05] J.-H. Lee, I.-Y. Jang, Y. S. Jeong, B. Seung, S.-Y. Moon, S.-G. Woo, H. K. Cho, SAMSUNG Electronics Co., Ltd. (Korea, Republic of)

737907 Etch characterization of binary mask dependence on mask material and resist thickness for 22nm mask fabrication [7379-06]

S. Nemoto, Toppan Photomasks, Inc. (United States); T. Faure, R. Wistrom, S. Crawford, G. Reid, P. Bartlau, IBM Corp. (United States); T. Komizo, Toppan Photomasks, Inc. (United States); A. E. Zweber, IBM Corp. (United States)

\section{MATERIAL AND PROCESS II}

737909 Effect of pellicle frame and adhesive material on final photomask flatness [7379-08] N. Zhou, K. Racette, D. Hasselbeck, M. Barrett, R. Nolan, M. Caterer, IBM Corp. (United States); T. Mizoguchi, S. Akutagawa, Toppan Photomasks, Inc. (Japan); G. Dickey, T. Shirasaki, ShinEłsu Chemical Co. Ltd. (Japan)

7379 OA Adhesion control between resist patterns and photomask blank surfaces [7379-09] M. Kurihara, Dai Nippon Printing Co., Ltd. (Japan) and Tokyo Institute of Technology (Japan); S. Hatakeyama, K. Yoshida, T. Nagai, D. Tołsukawa, M. Fukuda, Y. Morikawa, H. Mohri, M. Hoga, N. Hayashi, Dai Nippon Printing Co., Ltd. (Japan); H. Ohtani, M. Fujihira, Tokyo Institute of Technology (Japan)

$7379 \mathrm{OB} \quad$ Investigation of the development process for high-precision patterning [7379-10] J. Watanabe, T. Yamazaki, M. Tanabe, Toppan Printing Co., Ltd. (Japan); T. Komizo, Toppan Photomasks Inc. (United States); A. E. Zweber, A. C. Smith, IBM Corp. (United States)

7379 OD Study on surface integrity in photomask resist strip and final cleaning processes [7379-12] S. Singh, Hamatech USA Inc. (United States); S. Helbig, P. Dress, HamaTech APE (Germany); U. Dietze, Hamatech USA Inc. (United States) 
7379 OF Haze growth on reticles: What's the RigHT thing to do? [7379-14]

S. M. McDonald, D. V. Chalom, M. J. Green, J. A. McMurran, Photronics, Inc. (United States); M. B. Garrett, Micron Technology, Inc. (United States); D. W. Dlouhy, Photronics, Inc. (United States)

EUVL MASK I

7379 OG SEMATECH EUVL mask program status (Invited Paper) [7379-15]

H. Yun, F. Goodwin, S. Huh, K. Orvek, B. Cha, A. Rastegar, P. Kearney, SEMATECH, Inc. (United States)

$7379 \mathrm{OH} \quad$ Actinic EUVL mask blank inspection and phase defect characterization [7379-16] T. Yamane, T. Iwasaki, T. Tanaka, T. Terasawa, O. Suga, Semiconductor Leading Edge Technologies, Inc. (Japan); T. Tomie, National Institute of Advanced Industrial Science and Technology (Japan)

7379 OI Improvement of EUVL mask blank inspection capability at Intel [7379-17] A. Ma, T. Liang, S.-J. Park, G. Zhang, Intel Corp. (United States); T. Tamura, K. Omata, Y. Sato, H. Kusunose, Lasertec Corp. (Japan)

7379 0J Thorough characterization of an EUV mask [7379-18]

H. Mizuno, Toshiba America Electronic Components, Inc. (United States); G. Mclntyre, C. Koay, IBM Advanced Lithography research (Univted States); M. Burkhardt, IBM Corp. (United States); L. He, SEMATECH, Inc. (United States); J. Hartley, C. Johnson,

S. Raghunathan, College of Nanoscale Science and Engineering (United States);

K. Goldberg, I. Mochi, Lawrence Berkeley National Lab. (United States); B. La Fontaine, O. Wood, GLOBALFOUNDARIES (United States)

NIL AND PATTERNED MEDIA

7379 OK Nano-pattern design and technology for patterned media magnetic recording (Invited Paper) [7379-19]

H. Kataoka, Hitachi Global Storage Technologies Japan, Ltd. (Japan); Y. Hirayama, Hitachi, Ltd. (United States); T. R. Albrecht, M. Kobayashi, Hitachi Global Storage Technologies, Inc. (United States)

$7379 \mathrm{OL} \quad$ Si-mold fabrication for patterned media using high-resolution chemically amplified resist [7379-20]

M. Fukuda, T. Chiba, M. Ishikawa, K. Itoh, M. Kurihara, M. Hoga, Dai Nippon Printing Co., Ltd. (Japan)

7379 OM Trade-off between inverse lithography mask complexity and lithographic performance [7379-57]

B.-G. Kim, S. Suh, B.-S. Kim, S.-G. Woo, H.-K. Cho, SAMSUNG Electronics Co., Ltd. (Korea, Republic of); V. Tolani, G. Dai, D. Irby, K, Wang, G, Xiao, D. Kim, K.-H. Baik, B. Gleason, (Luminescent Technologies, Inc. (USA) 
$7379 \mathrm{ON}$ Inspection and repair for imprint lithography at $32 \mathrm{~nm}$ and below [7379-22]

K. Selinidis, E. Thompson, S. V. Sreenivasan, D. J. Resnick, Molecular Imprints, Inc. (United States); M. Pritschow, J. Butschke, M. Irmscher, H. Sailer, Institut für Mikroelektronik Stuttgart (Germany); H. Dobberstein, NaWoTec GmbH, Carl Zeiss SMT Co. (Germany)

\section{EUVL MASK II}

$737900 \quad$ Implications of image plane line-edge roughness requirements on extreme ultraviolet mask specifications (Invited Paper) [7379-23]

P. P. Naulleau, S. A. George, Lawrence Berkeley National Lab. (United States)

7379 OP Mask-induced aberration in EUV lithography [7379-24]

Y. Nakajima, T. Sato, R. Inanami, T. Nakasugi, T. Higashiki, Toshiba Corp. (Japan)

$73790 Q \quad$ Evaluation of an e-beam correction strategy for compensation of EUVL mask non-flatness [7379-25]

K. Orvek, J. Sohn, SEMATECH, Inc. (United States); J. Choi, SAMSUNG Electronics (United States); R. Engelstad, Univ. of Wisconsin, Madison (United States); S. Raghunathan, Univ. at Albany, SUNY (United States); J. Zimmerman, T. Laursen, ASML (United States); Y. Shusuke, NuFlare Technology, Inc. (United States); T. Shoki, HOYA Corp. (United States)

7379 OR Investigation of EUV mask defectivity via full-field printing and inspection on wafer [7379-26] R. Jonckheere, D. Van Den Heuvel, F. Iwamoto, N. Stepanenko, A. Myers, M. Lamantia, A.-M. Goethals, E. Hendrickx, K. Ronse, IMEC (Belgium)

\section{STRATEGY AND BUSINESS}

7379 OT Smart way to determine and guarantee mask specifications: trade-off between cost and quality (Invited Paper) [7379-28]

F. Shigemitsu, Toshiba Corp., Semiconductor Co. (Japan)

7379 OU Economics of automation for the design-to-mask interface [7379-29]

W. Erck, Wes Erck \& Associates (United States)

7379 OV Novel mask qualification methodology with die-to-database wafer inspection system [7379-30]

Y. Hagio, I. Nagahama, Y. Matsuoka, H. Mukai, K. Hashimoto, Toshiba Corp., Semiconductor Co. (Japan)

EDA, DFM, AND MDP

7379 OW European MEDEA+ CRYSTAL project: DFM photomasks inputs for EDA workflow task force (Invited Paper) [7379-31]

E. Beisser, XYALIS sarl (France); M. Tissier, Toppan Photomasks SAS (France); D. Au, Atmel Rousset SAS (France); S. Bonniol, Satin IP Technologies SAS (France); P. Garcia, Atmel Rousset SAS (France); P. Morey-Chaisemartin, XYALIS sarl (France); D. Sadran, Toppan Photomasks SAS (France); I. Servin, CEA, LETI (France); M. Tabusse, Satin IP Technologies SAS (France) 
7379 OX Reduction of MRC error review time through the simplified and classified MRC result [7379-32]

C. W. Lee, J. C. Lin, F. F. Chen, Toppan Chunghwa Electronics Corp. (Taiwan)

7379 OY Multi-core advantages for mask data preparation [7379-33]

J. Yeap, J. Nogatch, Synopsys, Inc. (United States)

$73790 Z$ GPU-accelerated inverse lithography technique [7379-34]

J. Zhang, Y. Deng, W. Xiong, Y. Peng, Z. Yu, Tsinghua Univ. (China)

737910 Effect of scanner illumination and lens transmittance signatures on OPC accuracy [7379-35] H.-T Huang, A. Sezginer, Cadence Design Systems, Inc. (United States); J. K. Tyminski, Nikon Precision Inc. (United States)

$737911 \quad$ Lithography compliance check considering neighboring cell structures for robust cell design [7379-36]

M. Miyairi, S. Nojima, S. Maeda, K. Kodera, R. Ogawa, S. Tanaka, Toshiba Corp.,

Semiconductor Co. (Japan)

\section{METROLOGY}

737912 The imaging performance of flash memory masks characterized with AIMS (Invited Paper) [7379-37]

E. van Setten, O. Wismans, K. Grim, J. Finders, ASML Netherlands B.V. (Netherlands); M. Dusa, ASML TDC (United States); R. Birkner, R. Richter, T. Scherübl, Carl Zeiss SMS GmbH (Germany)

737913 A new optical measurement method for verifying the exact change of thin films on the QZ blanks with ellipsometer [7379-73]

S. Moon, S.-Y. Kim, G.-Y. Bang, B.-G. Kim, S.-G. Woo, H. Cho, SAMSUNG Electronics Co., Ltd. (Korea, Republic of)

737914 Calibration strategies for precision stages in state-of-the-art registration metrology [7379-39] A. Huebel, U. Schellhorn, M. Arnz, G. Klose, Carl Zeiss SMT AG (Germany); D. Beyer, Carl Zeiss SMS GmbH (Germany)

737915 In-die mask registration metrology for 32nm node DPT lithography [7379-40]

K.-D. Roeth, F. Laske, M. Heiden, D. Adam, A. Boesser, KLA-Tencor GmbH (Germany); K. Rinn, A. Schepp, Univ. of Applied Sciences Giessen-Friedberg (Germany); J. Bender, KLA-Tencor GmbH (Germany)

737916 Phase behavior through pitch and duty cycle and its impact on process window [7379-41] U. Buttgereit, R. Birkner, D. Seidel, S. Perlitz, Carl Zeiss SMS GmbH (Germany); V. Philipsen,

P. De Bisschop, IMEC (Belgium)

\section{WRITING TECHNOLOGY}

737917 E-beam shot count estimation at $32 \mathrm{~nm} \mathrm{HP}$ and beyond [7379-42]

J. Choi, S. H. Lee, D. Nam, B. G. Kim, S.-G. Woo, H. K. Cho, SAMSUNG Electronics Co., Ltd.

(Korea, Republic of) 
737918 Present status of multi-column cell exposure system for mask writing [7379-43] H. Yasuda, A. Yamada, M. Yamabe, Association of Super-Advanced Electronics Technologies (Japan)

737919 Mask and wafer evaluation of Sigma7500 pattern generator applied to $65 \mathrm{~nm}$ logic metal and via layers [7379-44]

F. Liu, I. Shi, Q. Liu, L. Zhu, S. Zhao, E. Guo, Semiconductor Manufacturing International Corp. (China)

$73791 \mathrm{~A} \quad$ Reduction of resist charging effect by EB reticle writer EBM-7000 [7379-45]

M. Saito, K. Ugajin, O. Ikenaga, Toshiba Corp. (Japan)

\section{INSPECTION AND REPAIR I}

7379 1B Mask-LMC: lithographic simulation and defect detection from high-resolution mask images [7379-46]

G. Chen, J. N. Wiley, J.-S. Wang, R. C. Howell, S. Bai, Y.-F. Chen, F. Chen, Y. Cao, Brion Technologies, Inc. (United States); T. Takigawa, T. Kurosawa, Brion Technologies KK (Japan); H. Tsuchiya, K. Usuda, M. Tokita, NuFlare Technology, Inc. (Japan); F. Ozaki, N. Kikuiri, Y. Tsuji, Advanced Mask Inspection Technology, Inc. (Japan)

7379 1C Implementation strategy of wafer-plane and aerial-plane inspection for advanced mask manufacture [7379-47]

W.-S. Kim, D.-H. Chung, C.-U. Jeon, H. Cho, Samsung Electronics, Co. Ltd. (Korea, Republic of); W. Huang, J. Miller, G. Inderhees, B. Pinto, KLA-Tencor Corp. (United States); J. Hur, K. Park, J. Han, KLA-Tencor Korea (Korea, Republic of)

7379 1D Reticle inspection-based critical dimension uniformity [7379-77]

V. Vellanki, C. Hess, G. Pan, C. Chen, G. Inderhees, D. Lopez, KLA-Tencor Corp. (United States)

7379 IF Mask defect auto disposition based on aerial image in mask production [7379-50]

C. Y. Chen, L. Tuo, C. S. Yoo, Tawian Seminconductor Manufacturing Company (Taiwan);

L. Pang, D. Peng, J. Sun, Luminescent Technologies, Inc. (United States)

\section{INSPECTION AND REPAIR II}

7379 1G Airborne molecular contamination detection method for photomasks and ultra purging decontamination [7379-51]

H. Kambara, A. Favre, M. Davenet, Adixen by Alcatel-Lucent Co. (France); D. Rodier, Particle Measuring Systems, Inc. (United States)

$73791 \mathrm{H} \quad$ A new approach to reticle haze defect management in the fab [7379-52] Y.-D. Gau, K. Hsiao, W.-H. Hsu, Y.-M. Lu, Nanya Technology Corp. (Taiwan); C.-C. Chen, C. M. Liu, M. Van Riet, N. Gaspar, C.-C. Yu, P. Chan, KLA-Tencor Corp. (United States)

737911 Advances in post AFM repair cleaning of photomask with $\mathrm{CO}_{2}$ cryogenic aerosol technology [7379-53]

C. Bowers, I. Varghese, M. Balooch, W. Brandt, Eco-Snow Systems LLC, Linde AG (United States) 
7379 1 J Selective removal of persistent particles with no photomask damage [7379-54]

T. Robinson, R. Bozak, R. White, M. Archuletta, D. Lee, RAVE, LLC (United States)

MASK-RELATED LITHOGRAPHY

7379 IL Comparison of lithographic performance between MoSi binary mask and MoSi attenuated PSM [7379-56]

M. Yamana, Toppan Printing Co., Ltd. (Japan); M. Lamantia, Toppan Photomasks, Inc. (United States); V. Philipsen, IMEC (Belgium); S. Wada, T. Nagatomo, Y. Tonooka, Toppan Printing Co., Ltd. (Japan)

7379 1M Trade-off between inverse lithography mask complexity and lithographic performance [7379-57]

B.-G. Kim, S. S. Suh, B. S. Kim, S.-G. Woo, H. K. Cho, SAMSUNG Electronics Co., Ltd. (Korea, Republic of); V. Tolani, G. Dai, D. Irby, K. Wang, G. Xiao, D. Kim, K.-H. Baik, B. Gleason, Luminescent Technologies, Inc. (United States)

7379 iN Double patterning addressing imaging challenges for near- and sub- $k_{1}=0.25$ node layouts [7379-58]

B. -S. Seo, D.-K. Kang, M.-S. Noh, S.-H. Lee, SAMSUNG Electronics Co., Ltd. (Korea, Republic of); C. Cork, Synopsys SARL (France); G. LukPat, A. Miloslavsky, X. Li, K. Lucas, Synopsys, Inc. (United States); S. Lee, Synopsys Korea Inc. (Korea, Republic of)

737910 Novel OPC and DfM methodology for 3D memory device [7379-59]

T. Taguchi, T. Kotani, H. Mukai, H. Mashita, K. Iyanagi, K. Hashimoto, S. Inove, Toshiba Corp., Semiconductor Co. (Japan)

7379 IP High sensitivity electric field monitoring system for control of field-induced CD degradation in reticles (EFM) [7379-60]

T. Sebald, ESTION GmbH \& Co. KG (Germany); G. Rider, Microtome, Inc. (United States)

POSTER SESSION: MATERIAL AND PROCESS

$73791 Q \quad$ The art of photomask materials for low-k1-193nm lithography [7379-114]

M. Hashimoto, H. Iwashita, H. Mitsui, HOYA Corp. (Japan)

7379 IR Evaluation of the flatness effects of mask backing and orientation during photomask pellicle mount [7379-61]

T. Mizoguchi, S. Akutagawa, Toppan Photomasks, Inc. (United States); M. Barrett, M. Caterer, R. Nolan, K. Racette, D. Plouffe, N. Zhou, IBM Corp. (United States)

7379 is Evaluation for EAPSM life time by ArF pellicle characteristic [7379-62]

K. J. Seo, J. S. Ryu, G. M. Jeong, S. C. Kang, Y. D. Kim, S. C. Kim, C. Y. Kim, Hynix Semiconductor Inc. (Korea, Republic of)

7379 1T In situ selectivity monitor for dry etch of photomasks [7379-63]

T. Zhou, J. Chen, M. Grimbergen, M. Chandrachood, I. Ibrahim, A. Kumar, Applied Materials, Inc. (United States) 
7379 IU Plasma optical emission analysis for chamber condition monitor [7379-64]

Z. Mao, T. Zhou, M. Grimbergen, D. Bivens, D. Knick, R. Koch, M. Chandrachood, J. Chen,

I. Ibrahim, A. Kumar, Applied Materials, Inc. (United States)

7379 IV Fine pattern fabrication property of binary mask and attenuated phase shift mask [7379-65] T. Yamazaki, Y. Kojima, M. Yamana, T. Haraguchi, T. Tanaka, Toppan Printing Co., Ltd. (Japan)

7379 IW Applied analytics on EAPSM Cr plasma etch optimization utilizing design of experiment [7379-67]

M. J. Tian, E. Wang, Z. H. Zhu, Semiconductor Manufacturing International Corp. (China)

7379 1X Study of electric-field-induced-development method [7379-68]

M. Terayama, H. Sakurai, M. Sakai, M. Itoh, O. Ikenaga, Toshiba Corp. (Japan); H. Funakoshi, Tokyo Electron Kyushu Ltd. (Japan); T. Shiozawa, S. Miyazaki, Y. Saito, Tokyo Electron Ltd. (Japan); N. Hayashi, Dai Nippon Printing Co., Ltd. (Japan)

7379 IY Two-fluid cleaning technology for advanced photomask [7379-115]

T. Kikuchi, N. Kobayashi, Y. Kurokawa, H. Hirose, M. Nonaka, Shibaura Mechatronics Corp. (Japan)

\section{SESSION 16 POSTER SESSION: WRITING TECHNOLOGY}

737912 Improvement of data transfer speed and development of an EB data verification system in a VSB mask writer [7379-70]

O. Wakimoto, H. Manabe, H. Hoshi, N. Samoto, JEOL Ltd. (Japan) and Association of Super-Advanced Electronic Technologies (Japan; T. Komagata, Y. Nakagawa, JEOL Ltd. (Japan); M. Yamabe, Association of Super-Advanced Electronics Technologies (Japan)

737921 Quantification of electron-beam proximity effects using a virtual direct write environment [7379-72]

M. Schulz, Synopsys GmbH (Germany); P. Brooker, A. Zepka, G. Meyers, Synopsys, Inc. (United States)

\section{POSTER SESSION: METROLOGY}

737922 An evaluation of a new side-wall-angle measurement technique for mask patterns by CD-SEM [7379-38]

H. Hakii, I. Yonekura, M. Kawashita, Y. Kojima, Y. Sakamoto, K. Tanaka, Toppan Printing Co., Ltd. (Japan)

737924 CD performance evaluation according to advanced marking parameter [7379-75] S.-K. Yoon, S.-Y. Kim, K.-M. Yeon, C. Lim, S.-J. Choi, J.-H. Kim, L.-J. Kim, Y.-R. Cho, H.-S. Kim, Toppan Photomasks Korea, Ltd. (Korea, Republic of)

737925 Phase-shift/transmittance measurements in a micro pattern using MPM193EX [7379-76]

H. Nozawa, T. Ishida, S. Kato, O. Sato, K. Miyazaki, K. Takehisa, N. Awamura, H. Takizawa,

H. Kusunose, Lasertec Corp. (Japan) 
737927 An open-architecture approach to defect analysis software for mask inspection systems [7379-79]

M. Pereira, R. R. Pai, M. M. Reddy, R. M. Krishna, SoftJin Technologies Pvt. Ltd. (India)

\section{POSTER SESSION: REPAIRING TOOLS AND TECHNOLOGIES}

737928 The study of defect detection method for 32nm technology node and beyond [7379-80]

K. Seki, M. Shibita, S. Akima, Toppan Printing Co., Ltd. (Japan)

737929 Variable sensitivity detection (VSD) technology for screening SRAF nuisance defects [7379-81]

K. Yamashita, N. Harabe, M. Hirono, Y. Tamura, I. Isomura, Y. Tsuji, Advanced Mask Inspection Technology, Inc. (Japan); E. Matsumoto, NuFlare Technology, Inc. (Japan)

7379 2A A study of mask inspection method with pattern priority and printability check [7379-82] M. Tokita, H. Tsuchiya, T. Inove, T. Inove, M. Yamabe, Association of Super-Advanced Electronics Technologies (Japan)

7379 2B TeraScanXR: a high sensitivity and throughput photomask inspection system [7379-83] B. Mu, A. Dayal, A. Goonesekera, P. Lim, C. Chen, P. Liu, K. Yeung, B. Pinto, B. Broadbent, G. Inderhees, KLA-Tencor Corp. (United States)

7379 2C A noble evaluation method for repaired area utilizing SEM images [7379-84] K. Morishita, S. Kanamitsu, T. Hirano, Toshiba Corp., Semiconductor Co. (Japan)

7379 2D Semi-automated repair verification of aerial images [7379-85]

E. Poortinga, Carl Zeiss SMT Inc. (United States); T. Schereubl, R. Richter, Carl Zeiss SMS GmbH (Germany)

\section{POSTER SESSION: EUVL MASK}

7379 2G Novel absorber materials for EUV lithography mask [7379-88] T. Matsuo, K. Kanayama, Y. Okumoto, Toppan Printing Co., Ltd. (Japan)

$73792 \mathrm{H} \quad$ The impact of mask design on EUV imaging [7379-89] T. Schmoeller, Synopsys GmbH (Germany); J. K. Tyminski, Nikon Precision Inc. (United States); J. Lewellen, Synopsys Inc. (United States); W. Demmerle, Synopsys GmbH (Germany)

73792 Study of CD variation at EUV mask fabrication occurred by electric conduction from top to back side [7379-90]

K. Takai, K. Murano, K. Hagihara, M. Itoh, Toshiba Corp. (Japan); T. Abe, T. Adachi, H. Akizuki, T. Takikawa, H. Mohri, N. Hayashi, Dai Nippon Printing Co., Ltd. (Japan)

$73792 \mathrm{~J} \quad$ Actinic mask inspection using an extreme ultraviolet microscope [7379-91]

K. Takase, Y. Kamaji, T. Iguchi, Univ. of Hyogo (Japan); T. Sugiyama, T. Uno, Asahi Glass Co., Ltd. (Japan); T. Harada, T. Watanabe, H. Kinoshita, Univ. of Hyogo (Japan) 
7379 2K Evaluation of EUVL mask pattern defect inspection using 199nm inspection tool with super-resolution method [7379-92]

H. Shigemura, T. Amano, Y. Nishiyama, O. Suga, Y. Arisawa, MIRAI Semiconductor Leading Edge Technologies, Inc. (Japan); H. Hashimoto, K. Takahara, K. Usuda, NuFlare Technology, Inc. (Japan); N. Kikuiri, R. Hirano, Advanced Mask Inspection Technology, Inc. (Japan)

7379 2L FIB mask repair technology for EUV mask [7379-93]

T. Amano, Y. Nishiyama, H. Shigemura, T. Terasawa, O. Suga, MIRAI Semiconductor Leading Edge Technologies, Inc. (Japan); K. Shiina, F. Aramaki, A. Yasaka, SII NanoTechnology Inc. (Japan); T. Abe, H. Mohri, Dai Nippon Printing Co., Ltd. (Japan)

737920 Go proton: investigation on mask patterning for the $22 \mathrm{~nm}$ hp node using a ML2 multibeam system [7379-96]

J. Butschke, M. Irmscher, H. Sailer, Institut für Mikroelektronik Stuttgart Chips (Germany);

H. Loeschner, E. Platzgummer, Institut für Mikroelektronik Stuttgart Nanofabrication AG (Austria)

\section{POSTER SESSION: NIL AND PATTERNING MEDIA}

$73792 \mathrm{Q} \quad$ Developing quartz wafer mold manufacturing process for patterned media [7379-98]

T. Chiba, M. Fukuda, M. Ishikawa, K. Itoh, M. Kurihara, M. Hoga, Dai Nippon Printing Co., Ltd. (Japan)

7379 2S Progress of UV-NIL template making [7379-100]

T. Hiraka, J. Mizuochi, Y. Nakanishi, S. Yusa, S. Sasaki, Y. Morikawa, H. Mohri, N. Hayashi, Dai

Nippon Printing Co., Ltd. (Japan)

\section{POSTER SESSION: MASK-RELATED LITHOGRAPHY}

7379 2T Model-based assist feature insertion for sub-40nm memory device [7379-101]

S. Suh, S. Lee, S. Choi, S.-W. Lee, C. Park, SAMSUNG Electronics Co., Ltd. (Korea, Republic of)

POSTER SESSION: EDA, DFM, AND MDP

$73792 \mathrm{Y} \quad$ Optimizing computing resources for optimal throughput in a mask data preparation flow [7379-107]

W. Zhang, R. Bennett, P. Ghosh, S. Schulze, A. Bowhill, Mentor Graphics Corp. (United States)

$73792 Z \quad$ Model-based assist features [7379-108]

B. Yenikaya, O. Alexandrov, S. Chen, A. Liu, A. Mokhberi, A. Sezginer, Cadence Design Systems, Inc. (United States)

737930 Improvement of simulation accuracy using a non Gaussian kernel [7379-116] H. Futatsuya, T. Yamamoto, Fujitsu Microelectronics Ltd. (Japan); S. Yoshikawa, Fujitsu VLSI Ltd. (Japan); T. Chijimatsu, S. Asai, Fujitsu Microelectronics Ltd. (Japan) 
$737931 \quad$ Evaluation of mask data format standard OASIS.MASK developed for mask tools [7379-109] T. Suzuki, Dai Nippon Printing Co., Ltd. (Japan); Y. Nagaoka, Y. Maenaka, KLA-Tencor Corp. (United States); V. Vellanki, W. Ruch, KLA-Tencor Japan Ltd. (Japan); M. Mori, K. Hattori, K. Hosono, Renesas Technology Corp. (Japan); S. Narukawa, M. Hoga, H. Mohri, Dai Nippon Printing Co., Ltd. (Japan)

737932 Utilization of design intent information for mask manufacturing (II) [7379-110] K. Kato, M. Endo, T. Inove, M. Yamabe, Association of Super-Advanced Electronics Technologies (Japan)

737933 Study of the pattern categorization method in verification of OPC pattern [7379-1111] M. Naoe, T. Miyauchi, Fujitsu VLSI Ltd. (Japan); S. Makino, K. Suzuki, M. Oseki, T. Okada, Fujitsu Microelectronics Ltd. (Japan)

\section{POSTER SESSION: STRATEGY AND BUSINESS}

737934 Organized DFM [7379-112]

T. Sato, Toshiba Corp. (Japan); M. Honma, NEC Electronics Corp. (Japan); H. Itoh, Sharp Corp. (Japan); N. Iriki, Renesas Technology Corp. (Japan); S. Kobayashi, Toshiba Corp. (Japan); N. Miyazaki, Fujitsu Microelectronics Ltd. (Japan); T. Onodera, Oki Semiconductor Co., Ltd. (Japan); H. Suzuki, SANYO Semiconductor Co., Ltd. (Japan); N. Yoshioka, Fujitsu Microelectronics Ltd. (Japan); S. Arima, Univ. of Tsukuba (Japan); K. Kadota, National Institute of Advanced Industrial Science and Technology (Japan)

737935 Automated reticle inspection data analysis for wafer fabs [7379-113]

D. Summers, G. Chen, Freescale Semiconductor, Inc. (United States); B. Reese, T. Hutchinson, M. Liesching, H. Ying, R. Dover, KLA-Tencor Corp. (United States)

Author Index 


\title{
Symposium Committees
}

\author{
Symposium Chair
}

Toshiyuki Horiuchi, Tokyo Denki University (Japan)

Advisory Committee Chair

Yasuo Tarui, Tokyo University of Agriculture and Technology (Japan)

Advisory Committee

Masanori Komuro, New Energy and Industrial Technology

Development Organization (Japan)

Masatoshi Migitaka, Toyota Technological Institute (Japan)

Masao Otaki , Toppan Printing Company, Ltd. (Japan)

Norio Saitou, Nippon Institute of Technology (Japan)

Yoshio Tanaka, Luminescent Technologies, Inc. (Japan)

Organizing Committee Chair

Tohiyuki Horiuchi, Tokyo Denki University (Japan)

Organizing Committee Vice-Chair

Masato Shibuya, Tokyo Polytechnic Institute (Japan)

Organizing Committee

Uwe F. W. Behringer, UBC Microelectronics (Germany)

Parkson W. Chen, Taiwan Mask Corporation (Taiwan)

Hideaki Hamada, NuFlare Technology Inc. (Japan)

Naoya Hayashi, Dai Nippon Printing Company, Ltd. (Japan)

Kunihiro Hosono, Renesas Technology Corporation (Japan)

Masashi Iwatsuki, JEOL Ltd. (Japan)

Hiroichi Kawahira, Sony Corporation (Japan)

Masaomi Kameyama, Nikon Corporation (Japan)

Ichiro Mori, Semiconductor Leading Edge Technologies, Inc. (Japan)

Hiroaki Morimoto, Toppan Printing Company, Ltd. (Japan)

Osamu Nagarekawa, HOYA Corporation (Japan)

Kenichiro Okuda, SEMI Japan (Japan)

Patricia Marmillion, SEMATECH, Inc. (United States)

Hiroyuki Shigemura, Semiconductor Leading Edge Technologies, Inc. (Japan)

Yoshiki Suzuki, KLA-Tencor Japan Ltd. (Japan)

Tadahiro Takigawa, Brion Technologies KK (Japan)

Kochiro Tsujita, Canon, Inc. (Japan) 
J. Tracy Weed, Synopsys, Inc. (United States)

Masaki Yamabe, Association of Super-Advanced Electronics

Technologies (Japan)

Anto Yasaka, SII NanoTechnology Inc. (Japan)

Nobuyuki Yoshioka, Renesas Technology Corporation (Japan)

Steering Committee Chair

Hiroyuki Shigemura, Semiconductor Leading Edge Technologies, Inc.

(Japan)

Steering Committee Vice-Chairs

Morihisa Hoga, Dai Nippon Printing Company, Ltd. (Japan)

Tsuneo Terasawa, Semiconductor Leading Edge Technologies, Inc.

(Japan)

Steering Committee

Takayuki Abe, NuFlare Technology Inc. (Japan)

Naoya Hayashi, Dai Nippon Printing Company, Ltd. (Japan)

Kunihiro Hosono, Renesas Technology Corporation (Japan)

Naoyuki Ishiwata, Fujitsu Ltd. (Japan)

Ichiro Kagami, Sony Corporation (Japan)

Kokoro Kato, SII NanoTechnology Inc. (Japan)

Toshio Konishi, Toppan Printing Company (Japan)

Koichi Moriizumi, Lasertec Corporation (Japan)

Takeshi Nakajima, KLA-Tencor Japan Ltd.

Masatoshi Oda, NTT-AT Nanofabrication Corporation (Japan)

Yasushi Ohkubo, HOYA Corporation (Japan)

Toshiyuki Takahashi, JEOL Ltd. (Japan)

Hiroyoshi Tanabe, Intel Corporation (Japan)

Yoji Tonooka, Toppan Printing Company, Ltd. (Japan)

Hidehiro Watanabe, Toshiba Corporation (Japan)

Kazuo Yokoyama, Cadence Design Systems, Inc. (Japan) 


\title{
Conference Committee
}

\author{
Program Committee Chair
}

Kunihiro Hosono, Renesas Technology Corporation (Japan)

Program Committee Vice-Chairs

Kokoro Kato, SII NanoTechnology Inc. (Japan)

Toshio Konishi, Toppan Printing Company, Ltd.

Program Committee

Akihiko Ando, NEC Electronics Corporation (Japan)

Han-ku Cho, SAMSUNG Electronics Company Ltd. (Korea, Republic of)

Curt Jackson, Toppan Photomasks, Inc. (USA)

Jeff N. Frasworth, Intel Corporation (United States)

Thomas B. Faure, IBM Corporation (United States)

Takamitsu Furukawa, Oki Electric Industry Company, Ltd. (Japan)

Brian J. Grenon, Grenon Consulting, Inc. (United States)

Shigeru Hirukawa, Nikon Nippon Printing Company, Ltd. (Japan)

John Lin, Taiwan Semiconductor Manufacturing Company, Ltd.

(Taiwan)

Mark Ma, Photronics (United States)

Hiroshi Mohri, Dai Nippon Printing Company, Ltd. (Japan)

Koji Murano, Toshiba Corporation (Japan)

Yoshikazu Nagamura, Renesas Technology Corporation (Japan)

Yoshinori Nagaoka, KLA-Tencor Japan Ltd. (Japan)

Yasutoshi Nakagawa, JEOL Ltd. (Japan)

Nobuyuki Nishiguchi, Semiconductor Technology Academic Research

Center (Japan)

Tomoyuki Okada, Fujitsu Ltd. (Japan)

Hisatake Sano, Dai Nippon Printing Company, Ltd. (Japan)

Frank Schellenberg, Mentor Graphics Corporation (United States)

Yoshiyuki Sekine, Canon Inc. (Japan)

Yasunari Sohda, Hitachi Ltd. (Japan)

Kong Son, KLA-Tencor Corporation (United States)

Osamu Suga, Semidonductor Leading Edge Technologies, Inc.

(Japan)

Minoru Sugawara, Sony Corporation (Japan)

Yoji Takagi, Applied Materials Japan, Inc. (Japan)

Yoichi Usui, HOYA Corporation (Japan)

Hisashi Watanabe, Matsushita Electric Company, Ltd. (Japan) 
Session Chairs

Invited Session

Kunihiro Hosono, Renesas Technology Corporation (Japan)

John Lin, Taiwan Semiconductor Manufacturing Company Ltd.

(Taiwan)

Material and Process I

Toshio Konishi, Toppan Printing Company, Ltd. (Japan)

Koji Murano, Toshiba Corporation (Japan)

Thomas B. Faure, IBM Corporation (United States)

Material and Process II

Toshio Konishi, Toppan Printing Company, Ltd. (Japan)

Koji Murano, Toshiba Corporation (Japan)

Thomas B. Faure, IBM Corporation (United States)

EUVL Mask I

Osamu Suga, Semiconductor Leading Edge Technologies, Inc.

(Japan)

Rik M. Jonckheere, IMEC (Belgium)

NIL and Patterned Media

Morihisa Hoga, Dai Nippon Printing Company, Ltd. (Japan)

Hideo Kobayashi, HOYA Corporation(Japan)

EUVL Mask II

Yoshiyuki Sekine, Canon Inc. (Japan)

Patrick P. Naulleau, Lawrence Berkeley National Laboratory

(United States)

Strategy and Business

Kunihiro Hosono, Renesas Technology Corporation (Japan)

Uwe F. W. Behringer, UBC Microelectronics (Germany)

EDA, DFM, and MDP

Nobuyuki Nishiguchi, Semiconductor Technology Academic Research Center (Japan)

Tomoyuki Okada, Fujitsu Microelectronics Ltd. (Japan)

Frank M. Schellenberg, Mentor Graphics Corporation (United States)

Metrology

Hiroshi Mohri, Dai Nippon Printing Company, Ltd. (Japan)

Yoichi Usui, HOYA Corporation (Japan)

Han-Ku Cho, SAMSUNG Electronics Company, Ltd. (Korea, Republic of) 
Writing Technology

Yasutoshi Nakagawa, JEOL Ltd. (Japan)

Yasunari Sohda, Hitachi, Ltd. (Japan)

Inspection and Repair I

Akihiko Ando, NEC Electronics Corporation (Japan)

Yoshinori Nagaoka, KLA-Tencor Japan (Japan)

Inspection and Repair II

Yoshikazu Nagamura, Renesas Technology Corporation (Japan)

Christian Ehrlich, Carl Zeiss SMS GmbH (Germany)

Mask-related Lithography

Hisashi Watanabe, Panasonic Corporation (Japan)

Robert J. Naber, Cadence Design Systems, Inc. (United States) 
Downloaded From: https://www.spiedigitallibrary.org/conference-proceedings-of-spie on 26 Apr 2023

Terms of Use: https://www.spiedigitallibrary.org/terms-of-use 